\title{
Vicarious calibrations of HICO data acquired from the International Space Station
}

\author{
Bo-Cai Gao, ${ }^{1, *}$ Rong-Rong Li, ${ }^{1}$ Robert L. Lucke, ${ }^{1}$ Curtiss O. Davis, ${ }^{2}$ \\ Richard M. Bevilacqua, ${ }^{1}$ Daniel R. Korwan, ${ }^{1}$ Marcos J. Montes, ${ }^{1}$ \\ Jeffrey $\mathrm{H}$. Bowles, ${ }^{1}$ and Michael R. Corson ${ }^{1}$ \\ ${ }^{1}$ Remote Sensing Division, Naval Research Laboratory, Washington, DC 20375, USA \\ ${ }^{2}$ College of Earth, Ocean, and Atmospheric Sciences, Oregon State University, Corvallis, Oregon 97331, USA \\ ${ }^{*}$ Corresponding author: gao@nrl.navy.mil
}

Received 10 January 2012; revised 2 March 2012; accepted 5 March 2012;

posted 12 March 2012 (Doc. ID 161305); published 4 May 2012

\begin{abstract}
The Hyperspectral Imager for the Coastal Ocean (HICO) presently onboard the International Space Station (ISS) is an imaging spectrometer designed for remote sensing of coastal waters. The instrument is not equipped with any onboard spectral and radiometric calibration devices. Here we describe vicarious calibration techniques that have been used in converting the HICO raw digital numbers to calibrated radiances. The spectral calibration is based on matching atmospheric water vapor and oxygen absorption bands and extraterrestrial solar lines. The radiometric calibration is based on comparisons between HICO and the EOS/MODIS data measured over homogeneous desert areas and on spectral reflectance properties of coral reefs and water clouds. Improvements to the present vicarious calibration techniques are possible as we gain more in-depth understanding of the HICO laboratory calibration data and the ISS HICO data in the future. (C) 2012 Optical Society of America

OCIS codes: $\quad 010.0010,010.4450,120.0280,280.4788$.
\end{abstract}

\section{Introduction}

The Naval Research Laboratory (NRL) Hyperspectral Imager for the Coastal Ocean (HICO) instrument is the first space-borne imaging spectrometer specifically designed for coastal maritime imaging. A typical HICO scene is approximately $42 \mathrm{~km}$ cross-track (at nadir) and $192 \mathrm{~km}$ along track. Within a scene, the size of each picture element (pixel) on the ground is 90 by $90 \mathrm{~m}$. A detailed description of the HICO mission and instrument is given by Lucke et al. [1]. The HICO sensor, as part of an experimental payload, was launched [2] into space onboard the Japanese Aerospace Exploration Agency H-IIB Heavy-Lift Vehicle on September 11, 2009. It was transferred to its operating location on the Japanese Experiment Module-Exposed Facility on the Inter-

$1559-128 \mathrm{X} / 12 / 142559-09 \$ 15.00 / 0$

(C) 2012 Optical Society of America national Space Station (ISS), and data collection was initiated on September 25, 2009. The sensor is still operational at present.

Extensive prelaunch spatial, spectral, and radiometric characterization and calibration [1] of the $\mathrm{HICO}$ instrument was performed at the NRL in Washington, DC. However, HICO is not equipped with any onboard radiometric or spectral calibration monitoring sources. Therefore, we have relied on several vicarious calibration techniques for postlaunch monitoring and calibration, including spectrummatching of atmospheric gaseous absorption bands for wavelength calibration and intersatellite data comparisons for radiometric calibration. Previously, a vicarious calibration technique applicable for processing multichannel ocean color sensor data was described by Franz et al. [3]. A specific radiative transfer model for predicting the top-of-atmosphere (TOP) radiances is tied to the technique. In this paper, we describe the vicarious calibration techniques 
that have been used in converting the HICO hyperspectral L1A digital numbers (DNs) to the L1B radiometrically calibrated radiances.

\section{Spectral Calibration}

As described by Lucke et al. [1], the HICO spectral range is 0.35 to $1.08 \mu \mathrm{m}$ with the light dispersed over 384 pixels, giving an intrinsic high-resolution spectrum with a spectral sampling interval of $1.91 \mathrm{~nm}$ and a spectral resolution of approximately $3.2 \mathrm{~nm}$. However, in nominal operations, a three-to-one onboard binning is employed in the spectral direction, producing a spectral sampling of $5.73 \mathrm{~nm}$ per bin. Figure 1(a) shows an example of a HICO Level 1A (L1A) spectrum, in digital counts or DNs, collected in the nominal operational mode. The atmospheric water vapor bands centered near 0.725 , 0.825 , and $0.94 \mu \mathrm{m}$, the oxygen band near $0.765 \mu \mathrm{m}$, and a solar line near $0.430 \mu \mathrm{m}$, are clearly seen in the spectrum. These atmospheric and solar features allow for the determination of spectral resolution and wavelength calibration from the HICO data themselves. Figure 1(b) shows a sample HICO $\mathrm{DN}$ spectrum collected in the unbinned high-spectral resolution mode. Several solar lines below $0.8 \mu \mathrm{m}$, such as those near $0.430,0.485,0.517$, and $0.656 \mu \mathrm{m}$, are resolved much better in this spectrum than those in the Fig. 1(a) spectrum. It should be pointed out that some of the high frequency features above $0.8 \mu \mathrm{m}$ in the Fig. 1(b) spectrum are not real atmospheric or solar features. Rather, as described in Subsection 3D, these can be attributed to spectral

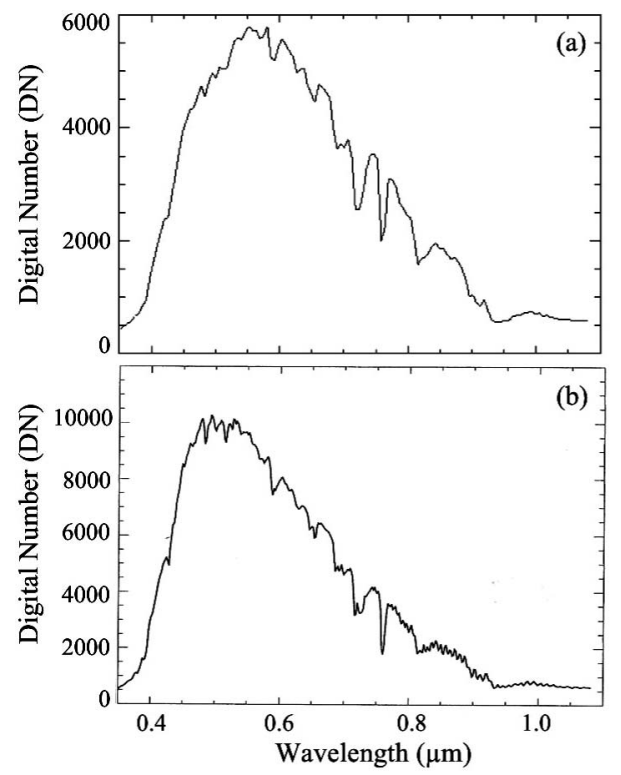

Fig. 1. (a) Sample HICO Level $1 \mathrm{~A}$ spectrum in digital numbers acquired in the normal data collection mode. (b) Similar to (a) except that the data were collected in the special high-spectral resolution mode. The atmospheric water vapor bands, centered near $0.725,0.825$, and $0.94 \mu \mathrm{m}$, the oxygen band near $0.765 \mu \mathrm{m}$, and a solar line near $0.430 \mu \mathrm{m}$, were observed in both spectra. etaloning [4]. A technique for mitigating the effect of this etaloning is also described in Subsection 3D.

Spectral matching techniques [5] were developed in the 1970s for retrieving line positions and line widths of atmospheric gases from laboratory-measured "ultra" spectra (in which individual lines in gas absorption bands are resolved). The retrieval accuracies [5] for line positions and widths were determined to be $1 \%$ and $2.5 \%$, respectively, of the spectral resolution. Similar spectral matching techniques [5] have been used in the estimates of channel positions and spectral resolution from airborne imaging spectrometer data. Because atmospheric gas absorption features are very sharp in the spectral domain, a spectral calibration accuracy for channel positions of approximately $1 \%$ of the spectral resolution is required [6] for the derivation of land or ocean surface reflectances from measured imaging spectrometer data. We use the spectrum-matching technique of Gao et al. [6] for on-orbit monitoring of the HICO spectral calibration. For this purpose, the oxygen A-band, located at $0.765 \mu \mathrm{m}$, is an ideal choice. The band is strong and, as Fig. 1 shows, well resolved in the HICO data. In addition, the variation with time or image location of the atmospheric oxygen column density is exceedingly small. Several atmospheric water vapor bands are also well resolved with HICO. However, these water vapor bands are not ideally suited for calibration purposes because of large temporal variations ( $>1$ order of magnitude) in the water vapor column density. Furthermore, under cold dry conditions, the water vapor absorption bands are too weak to be used for spectral calibration purposes.

As an example of the spectral matching technique applied to the HICO data, Fig. 2(a) shows a HICO spectrum, obtained from an early HICO scene, covering the $0.765 \mu \mathrm{m}$ oxygen band absorption region, and a theoretically simulated atmospheric gas transmittance spectrum. When plotting the HICO spectrum, the wavelength file from prelaunch laboratory calibrations was used. From this figure, it is seen that the oxygen bands in the measured and simulated spectra are shifted, indicating a postlaunch HICO wavelength shift.

In order to quantify the wavelength shift, we shifted the wavelengths of the HICO channels from $-3 \mathrm{~nm}$ to $+3 \mathrm{~nm}$ (relative to the prelaunch laboratory-calibrated wavelengths) in steps of $0.02 \mathrm{~nm}$. At each step, we performed a nonlinear least-squares matching between the measured spectrum and the simulated spectrum. During the matching process, the spectral background and the oxygen band transmittance for the calculated spectrum were automatically adjusted to minimize the difference between the measured and the simulated spectra. The sum of the squared differences between the two spectra at each step was calculated. Figure 2(b) shows the match between the measured spectrum (solid line) and the simulated spectrum (dotted line) when the relative shift between the two spectra was $-1.72 \mathrm{~nm}$. The 

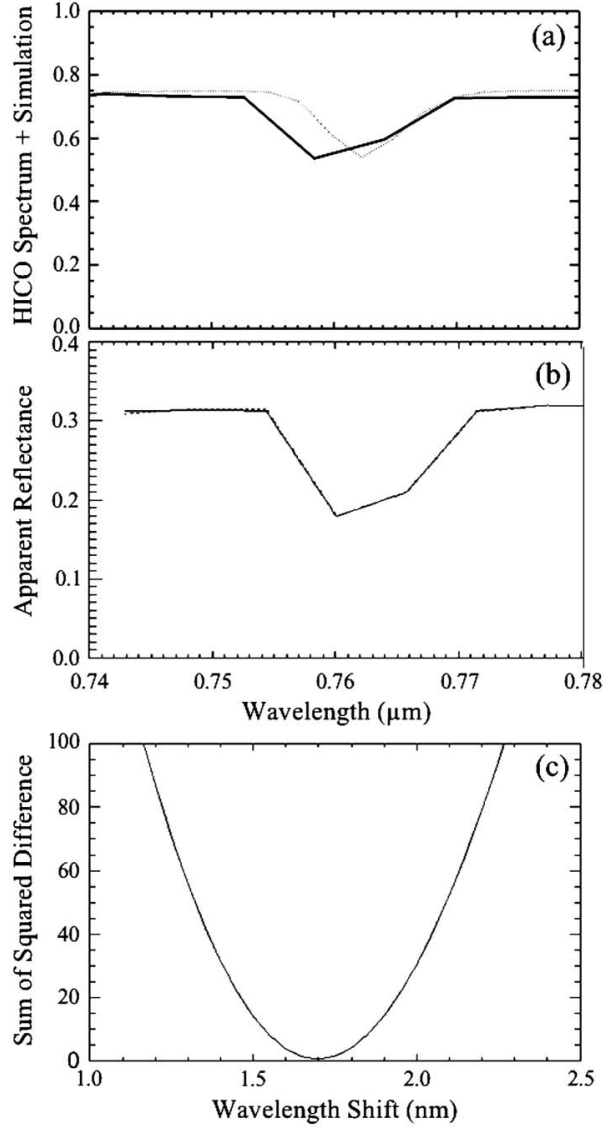

Fig. 2. (a) Example of ISS HICO spectrum (solid line) covering the $0.765 \mu \mathrm{m}$ oxygen band absorption region based on the wavelength table obtained from the prelaunch laboratory calibrations and a theoretically simulated atmospheric gas transmittance spectrum (dotted line). (b) Similar spectrum matching but with the wavelengths of the measured HICO spectrum being shifted by $1.72 \mathrm{~nm}$ to the right. (c) Sum of squared differences between the measured and fitted spectra as a function of wavelength shift. The minimum occurs for a wavelength shift of $\sim 1.7 \mathrm{~nm}$.

two spectra coincided almost exactly. Figure 2(c) is a plot of the sum of the squared differences as a function of wavelength shift. The minimum occurred at a wavelength shift of $1.72 \mathrm{~nm}$. This is considered to be the best estimate of wavelength shift for the HICO instrument soon after launching into space in September of 2009. The spectral alignment continued to shift rapidly for the first 100 days as the instrument adjusted to the space environment. But after that initial time, the rate of change in the spectral calibration was much lower. At present, the shift is relatively stable at approximately $0.9 \mathrm{~nm}$.

Figure 3(a) shows examples of HICO wavelength shifts in the cross-track direction from four sets of HICO data acquired on September 25, 2009; December 19, 2009; February 4, 2010; and September 8, 2011. For each curve, the amounts of shifts in the cross-track direction are not constant. On average, the difference in the amount of shift from the most left pixel to the most right pixel is about $0.5 \mathrm{~nm}$. This is consistent with the amount of spectral tilt
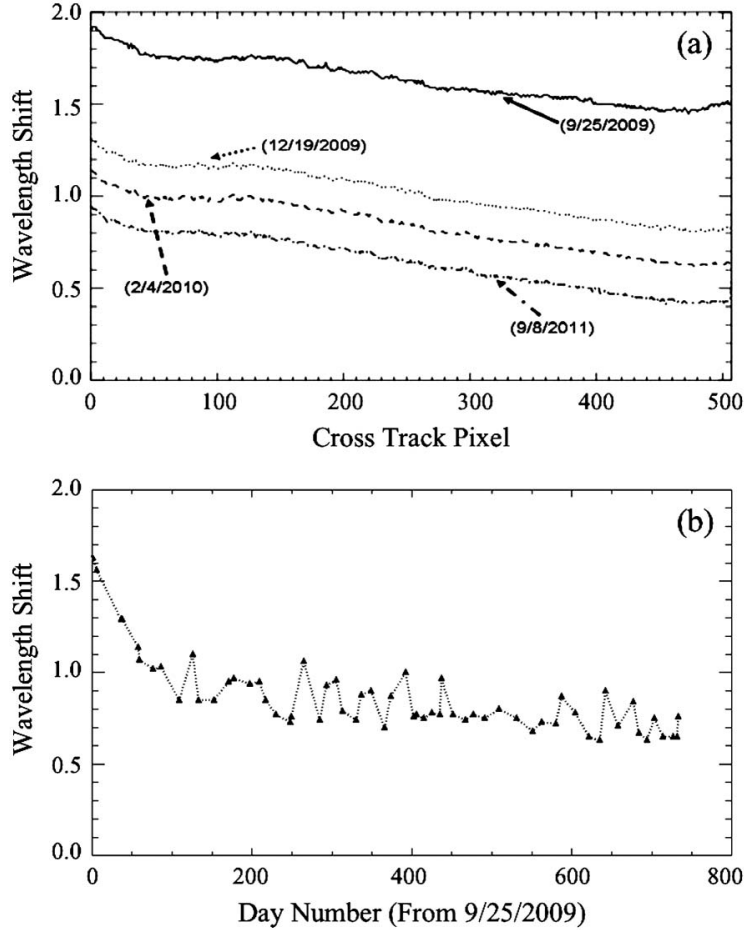

Fig. 3. (a) HICO wavelength shifts in the cross-track direction from four sets of HICO data acquired on September 25, 2009; December 19, 2009; February 4, 2010; and September 8, 2011. (b) Wavelength shift as a function of day number (from September 25, 2009) for a spatial pixel located at the center of the HICO focal plane array.

( $\sim .4 \mathrm{~nm}$ ) obtained from the HICO prelaunch laboratory calibration data as reported by Lucke et al. [1].

Among the four curves in Fig. 3(a), the curve (solid line) for September 25, 2009, the first day of HICOacquired scientific data from the ISS, shows the largest spectral shifts ( $\sim 1.7 \mathrm{~nm}$ at the array center) relative to the wavelengths of HICO prelaunch laboratory spectral calibrations. The shifts for the December 19, 2009, curve (dotted line) decreased to about $1.1 \mathrm{~nm}$. The shifts for the February 4, 2010, curve (dashed line) were approximately $0.9 \mathrm{~nm}$, and for the September 8, 2011, curve (dash-dotted line), the shift was about $0.7 \mathrm{~nm}$.

In order to investigate the evolution of wavelength shift with time in more detail, we have selected a total of 60 HICO datasets measured over clear land areas during the first 2 years of ISS HICO operations, beginning on September 25, 2009. Figure 3 (b) shows a plot of wavelength shift as a function of day number for a spatial pixel located at the center of the HICO focal plane array. It is seen that the wavelength shift decreased rapidly with time during the initial 100 days. Thereafter, the wavelength shift stabilized, indicating the stabilization of the HICO instrument. However, the wavelength shifts after the initial 100 days still had small fluctuations around a straight line with a slope of $-0.0004 \mathrm{~nm} /$ day, a vertical offset of $0.928 \mathrm{~nm}$, and a variance of 0.39. This slope is insignificant. Currently, we 
do not correct for these small fluctuations, and an offset of $0.9 \mathrm{~nm}$ was used in processing the HICO data after Day 100.

After the quantification of wavelength shift using the spectrum matching technique, as illustrated in Figs. 2(b) and 2(c), the spectral resolution of HICO data was determined using the same technique. Figure 4 shows a curve of the sum of squared difference as a function of full width at half maximum (FWHM) by fitting an atmospheric water vapor absorption band centered near $0.725 \mu \mathrm{m}$ in a HICO spectrum collected under the normal operational mode. The minimum occurred at a FWHM of $5.1 \mathrm{~nm}$, which is considered to be the best estimate of the FWHM of the HICO instrument. The FWHM remained the same after HICO was launched into space.

\section{Radiometric Calibration}

\section{A. Second-Order Light Corrections}

HICO does not contain a filter to block second-order light from the grating spectrometer $[1,7]$. Therefore, within the HICO spectral range of $0.3 \overline{5}$ to $1.08 \mu \mathrm{m}$, the second-order light in the wavelength interval from 0.35 to $0.54 \mu \mathrm{m}$ fell on the same pixels as the first-order light in the 0.7 to $1.08 \mu \mathrm{m}$ wavelength interval. In order to make radiometric calibrations of the near-infrared (NIR) channels, the second-order light contribution needs to be corrected. Through analysis of HICO imaging data containing features of shallow underwater objects, such as coral reefs, an empirical but effective method to correct for the second-order light effects has been developed and reported by Li et al. [7]. A brief description of the method is given below.

The method is empirical in nature and based on the fact that if the second-order light effects were not present, spatial features of coral reefs and other objects in shallow water areas should not be observed in narrow channel images at wavelengths above $0.8 \mu \mathrm{m}$. This is indeed the case for many sets of hyperspectral imaging scenes acquired with the NASA Airborne Visible Infrared Imaging Spectrometer (AVIRIS) [8] over Hawaiian waters with subsurface coral reefs in the spring of 2000 . The reason is that the solar radiation above $0.8 \mu \mathrm{m}$ is totally absorbed

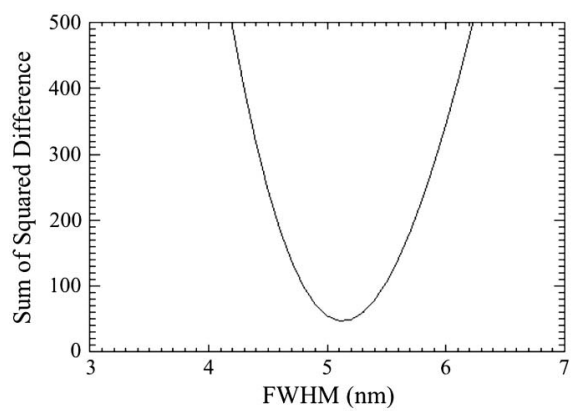

Fig. 4. Sum of squared differences between the measured and fitted spectra as a function of FWHM. The minimum occurs at a FWHM of $5.1 \mathrm{~nm}$. (a)

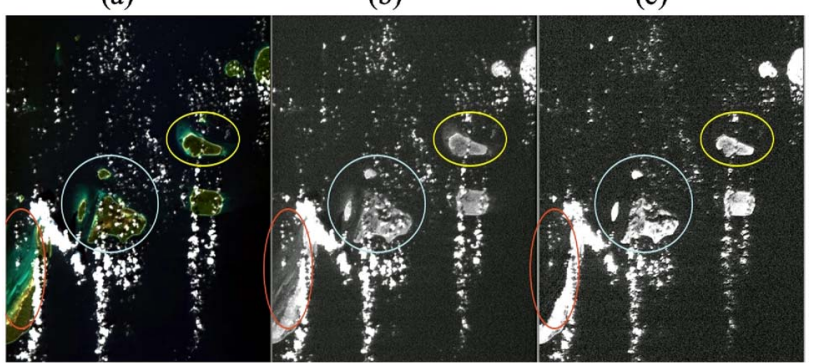

Fig. 5. (Color online) True color image (a) over the Philippine Sea on October 26, 2009, with features under shallow waters, the corresponding $1.0 \mu \mathrm{m}$ single channel image before (b) and after (c) the second-order light corrections.

by water in the first $2 \mathrm{~m}$ due to the large liquid water absorption [9]. Figure 5(a) shows a sample true color HICO image (red: $0.63 \mu \mathrm{m}$, green: $0.55 \mu \mathrm{m}$, and blue: $0.47 \mu \mathrm{m}$ ) acquired over the Philippine Sea on October 26, 2009. Shallow underwater features (greenish) around the islands are seen. Figure $5(\mathrm{~b})$ is the $1.0 \mu \mathrm{m}$ single channel image before the removal of second-order effects. Weak shallow water features remain in this image. They should not be visible because of strong liquid water absorption at $1 \mu \mathrm{m}$. The observation of spatial features of shallow water objects in the $1 \mu \mathrm{m}$ channel image is an indication of the presence of the second-order light effects.

For the description of the empirical method, we denote a shallow water spectrum as $S(\lambda)$ and a nearby deep water spectrum as $D(\lambda)$ in a HICO ISS dataset. We use $f(\lambda)$ to represent the empirical correction curve for removing the second-order light effects. The basic equation for the derivation of $f(\lambda)$ is

$$
S(\lambda)-f(\lambda) * S(\lambda / 2)=D(\lambda)-f(\lambda) * D(\lambda / 2),
$$

where $S(\lambda)$ is the signal (in DNs) from the shallow water area at wavelength $\lambda, D(\lambda)$ is the signal from the deep water area at $\lambda, S(\lambda / 2)$ is the signal from the shallow water area at half wavelength $(\lambda / 2)$, $D(\lambda / 2)$ is the signal from the deep water area at $(\lambda / 2)$, and $f(\lambda)$ is the empirical scaling factor for correcting the second-order light effect. $f(\lambda)$ is actually the fraction of the first-order light falling on the NIR detectors. The term, $f(\lambda) * S(\lambda / 2)$, on the left side of Eq. (1), is the second-order signal at the NIR wavelength $\lambda$ contributed by the signal at $\lambda / 2$ for the shallow water spectrum. Similarly, the term, $f(\lambda) * D(\lambda / 2)$, on the right side of Eq. (1), is the second-order signal for the deep water spectrum. After the corrections of the second-order effects, the NIR channel signal over the shallow water area and the deep water area should be equal. Eq. (1) implicitly assumes that both the water optical properties over the shallow water area and the nearby deep water area and the atmospheric conditions above these two areas are the same. These assumptions are expected to be valid over remote oceanic areas far away from land masses. Solving Eq. (1) for $f(\lambda)$, we obtain 


$$
f(\lambda)=[S(\lambda)-D(\lambda)] /[S(\lambda / 2)-D(\lambda / 2)]
$$

The wavelength dependence of $f(\lambda)$ for HICO has been given by Li et al. [7].

Now we use $A(\lambda)$ to represent the signal of an arbitrary pixel in a HICO scene after the removal of dark current and smear effects [1], and $C(\lambda)$ is the secondorder light corrected signal for the corresponding pixel. The equation for the second-order light correction is

$$
C(\lambda)=A(\lambda)-f(\lambda) * A(\lambda / 2)
$$

Figure $\underline{5(\mathrm{c})}$ is the second-order-light-corrected $1 \mu \mathrm{m}$ channel image for the scene of the Philippine Sea. The shallow water features around the island in this image are mostly removed, demonstrating that the second-order light effect is properly corrected.

\section{B. Derivation of a Constant Scaling Factor Based on Intersatellite Data Comparison}

Soon after the ISS HICO data became available, the calibration coefficients obtained from the prelaunch laboratory measurements were applied to the ISS HICO data to derive the calibrated L1B spectral radiances. Through preliminary analysis of the data, we realized that the radiance values were biased low. For example, when deriving water leaving reflectances from these spectra using a hyperspectral atmospheric correction algorithm [10], the retrieved waters leaving reflectances in the visible were negative, even if only Rayleigh scattering was included in atmospheric correction. This suggests that the radiometric sensitivity of the HICO instrument decreased significantly after launch.

Because the HICO sensor does not include an onboard radiometric calibration device, vicarious techniques were required for postlaunch calibration. For this purpose, we relied on intercomparisons between HICO hyperspectral data and multichannel data acquired with the NASA Terra and Aqua Moderate Resolution Imaging SpectroRadiometer (MODIS) [11] instruments. Initially, we tried to use $\mathrm{HICO} /$ MODIS data pairs over the Lanai Marine Optical Buoy (MOBY) [12] site in Hawaii. We realized that the MOBY site was frequently covered by moving cirrus clouds and time-varying air masses (from different sources). The MOBY site is too close to the nearby islands (mountains). We were unable to find any good clear HICO MODIS imaging pairs over the MOBY site with the same air mass. As a result, we decided not to use HICO/MODIS data pairs over the MOBY site for intersatellite data comparisons. Fortunately, although HICO is an ocean color instrument, it does not saturate over high-reflectivity desert areas. This permitted the use of a number of stable desert calibration sites previously used by the Landsat Science Team, the NASA EO-1 Science Team, and the NASA
JPL AVIRIS Team for intersatellite data comparisons. The calibration sites used included the Ivanpah Playa in California, Rogers Dry Lake in California, Bahrain in the Middle East, and Taklamakan Desert in Western China. A dozen pairs of HICO and MODIS images were acquired over these calibration sites under very clear atmospheric conditions on the same day with time differences of less than 1 hour between the measurements of the corresponding data pairs.

Figure 6 shows an example of such comparisons between $\bar{H}$ ICO data and MODIS data. Figure $\underline{6(a)}$ is a HICO true color image acquired over an area in the Taklamakan Desert on November 21, 2009. Figure $6(\mathrm{~b})$ is a Terra MODIS image acquired on the same day. Small, spatially uniform common areas were selected in both images, and the radiance spectra were averaged over these areas. Figure 6(c) shows the HICO and MODIS radiances as a function of wavelength. HICO has contiguous spectral coverage, while the MODIS has only a few discrete narrow channels in the 0.4 to $1.0 \mu \mathrm{m}$ spectral range. The line connecting the MODIS data points only serves as a guide to the eye. From this figure, it is seen that the radiances of the HICO channels are much smaller $(\sim 25 \%)$ than those of the corresponding MODIS channels. Through a number of comparisons between HICO data and MODIS data near 0.66 and $0.86 \mu \mathrm{m}$, we obtained scaling factors varying between 1.26 and 1.38. The variations are probably related to a variety of factors, including the surface bidirectional reflectance factors and polarization effects with both the

(a)

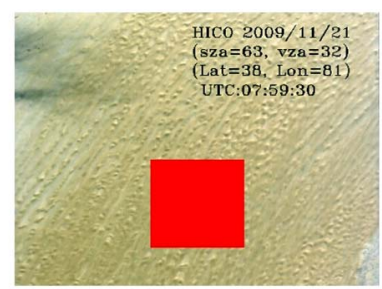

(b)

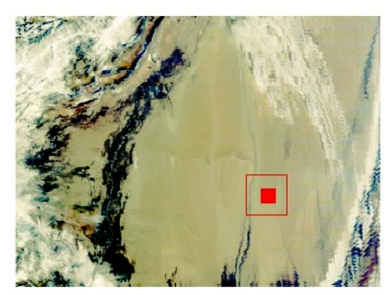

(c)

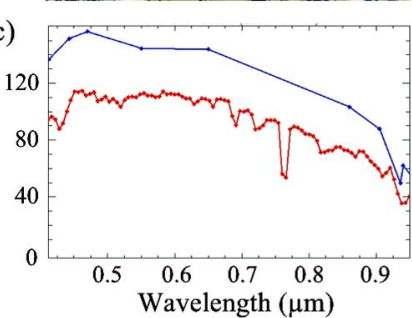

Fig. 6. (Color online) HICO image (a) and a MODIS image (b) acquired on November 21, 2009, over an area in the Taklamakan Desert; average radiance curves (c) for HICO data and MODIS data over the marked red rectangular areas in (a) and (b). 
HICO and MODIS instruments. Work to resolve these complex confounding factors is in progress. However, for the first release of the HICO data, we simply averaged the scaling factor obtained from processing all of the HICO/MODIS coincidences. This yielded a scaling factor of 1.32 , which was applied to each image in the L1B processing.

To date, the mechanism for the postlaunch loss of radiometric sensitivity in the HICO sensor has not been identified. We did suspect that the surface areas of the HICO front optical lens might be contaminated in the ISS environment. Therefore, arrangements were made to take high spatial resolution digital camera images of the HICO lens surface areas using one of the external manipulating arms on ISS. The resulting digital camera images demonstrated that the HICO lens surface appeared to be perfectly clean. Thus, at present, HICO's postlaunch loss of radiometric sensitivity remains a mystery.

\section{Additional Scaling Curve Based on Cloud Spectral Modeling}

After application of the constant scaling factor of 1.32 to the HICO L1B radiances, we began the evaluation of wavelength dependence of HICO radiances. This was accomplished by first converting the HICO L1B radiances into the satellite level "apparent reflectances," and then comparing the HICO apparent reflectance spectra over white clouds with those from theoretical simulations. The apparent reflectance $\rho *$ of a given channel is traditionally defined as

$$
\rho *=\pi L /\left(\mu_{0} E_{0}\right)
$$

where $L$ is the measured radiance, $\mu_{0}$ is the cosine of the solar zenith angle and $E_{0}$ is the extraterrestrial solar flux. Figure 7 shows an example of such a comparison. Figure $7(\overline{\mathrm{a}})$ is a portion of the HICO true color image acquired over an area near Midway Island in the Pacific Ocean on October 20, 2009. Many small white cumulus clouds are seen. The solid line in Fig. 7 (b) is a HICO apparent reflectance spectrum of a cloud pixel after application of the derived wavelength independent scaling factor of 1.32 . Over the visible and near-IR wavelength range of the HICO sensor, the reflectance of the cloud itself should be wavelength independent because typical water cloud particle sizes $(\sim 15 \mu \mathrm{m})$ are much larger than the observation wavelengths. Because of the presence of ozone, water vapor and oxygen above the cloud, the broad ozone absorption feature centered near $0.6 \mu \mathrm{m}$, narrower water vapor absorption bands centered near $0.725,0.825$, and $0.94 \mu \mathrm{m}$, and an oxygen band near $0.76 \mu \mathrm{m}$ are seen clearly. In addition, Rayleigh scattering effects above the cloud, which increase with decreasing wavelength, should result in an increase in the apparent reflectance with decreasing wavelength in cloudy pixels, primarily shortward of $0.45 \mu \mathrm{m}$. However, Fig. 7(a) shows a rapid decrease in apparent reflectance as wavelength decreases below $0.45 \mu \mathrm{m}$. This is likely due to errors
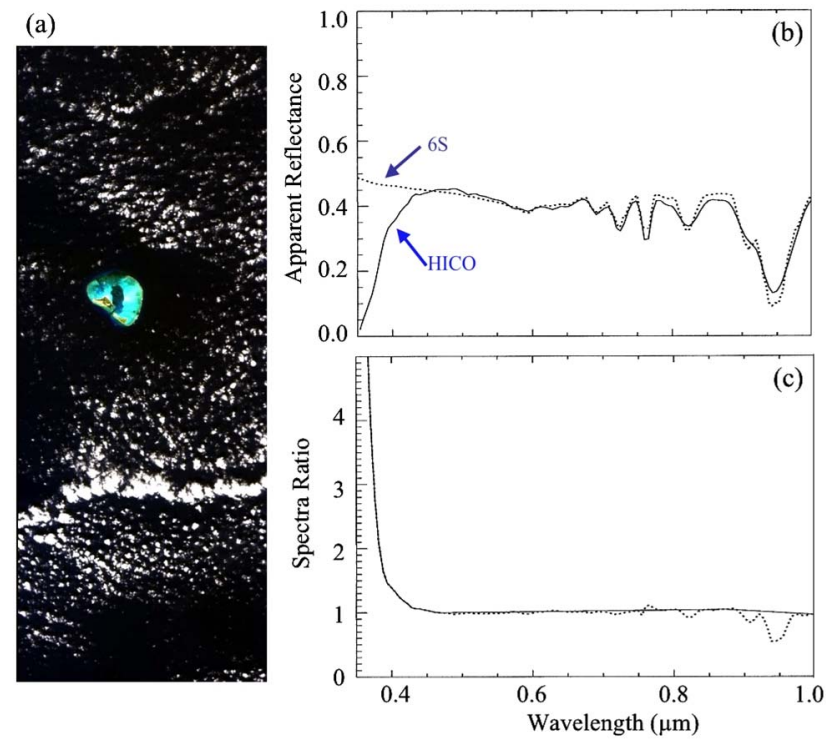

Fig. 7. (Color online) (a) HICO true color image acquired over an area around the Midway Island in the Pacific ocean on October 20, 2009. (b) HICO apparent reflectance spectrum (solid line) for a cloud pixel and the corresponding simulated cloud apparent reflectance spectrum (dashed line). (c) Mean (dashed line) of ratio spectra (simulated data over HICO data) and the derived scaling curve (solid line).

in laboratory radiometric calibration of the HICO instrument. It is often very difficult to make accurate radiometric calibrations of optical instruments in the blue, since standard radiation sources (at a temperature of $\sim 2,000 \mathrm{~K}$ ) do not emit a sufficient amount of photons in the blue.

In order to estimate the wavelength-dependent radiometric calibration errors evident in the solid line in Figure 7(b), we have simulated a TOA cloud apparent reflectance spectrum using the $6 \mathrm{~S}$ [13] radiative transfer code. This is given as the dashed line in Fig. 7(b). In the simulation, the estimated bottom cloud reflectance value, the cloud height, and a standard model atmosphere for ozone and water vapor amounts above the cloud were used. The shape of the simulated spectrum was qualitatively very similar to those measured with the European GOME (Global Ozone Monitoring Experiment) [14] satellite instrument. At wavelengths longer than $\overline{0.45} \mu \mathrm{m}$, the HICO measurement and the simulated reflectance spectrum were in excellent agreement, but below this wavelength, the HICO spectrum was increasingly biased low. In order to correct for the evident radiometric bias below $0.45 \mu \mathrm{m}$, we repeated the procedure illustrated in Figs. 7(a) and 7(b) for many other cloud scenes over the course of the mission and, in each case, calculated the ratio between the simulated and observed reflectance spectrum. The mean of the ratio spectra is shown as the dashed line in Fig. 7(c). Between 0.45 and $0.86 \mu \mathrm{m}$ and over atmospheric window regions (where the absorption by atmospheric gases are negligible), the ratio values are nearly constant and equal to 1 . Over atmospheric gaseous absorption regions, the ratio values deviate 
from 1 mainly due to errors in the assumed abovecloud water vapor and ozone column density used in the simulations. The solid line in Fig. 7(c) was obtained by selecting the data points in atmospheric window regions from the dashed line and interpolating across the gaseous absorption regions of the spectrum. In the L1B data processing, the scaling factor spectrum shown in Fig. 7(c) (the solid line) was applied to each HICO spectrum.

\section{Spectral Smoothing}

Figure 8 shows an example HICO scene obtained after application of the radiometric calibration procedures described in Subsection 3A to 3C (secondorder correction, wavelength-independent, and wavelength-dependent scaling). Figure 8(a) is a truecolor HICO image acquired over the mouth of the Chesapeake Bay on October 7, 2009. The radiance spectrum for a sandy area in the image in unit of $\mathrm{W} /\left(\mathrm{m}^{2} \mathrm{sr} \mu \mathrm{m}\right)$ multiplied by the number 50 is shown as the red line in Fig. 8(b).

High-frequency oscillations are prominent in the spectral region above $0.8 \mu \mathrm{m}$ [also see the spectrum in Fig. 1(b)]. These oscillations are attributed to spectral etaloning effects resulting from the use of a backilluminated charge-coupled device (CCD) in the HICO Focal Plane Array. The selection of a back-illuminated CCD for use in HICO was for the purpose of increasing sensitivity in the blue spectral region. However, reflections between the front and back surfaces of the CCD made them act as partial etalons ${ }^{3}$. The etalon-like property leads to undesired fringes of constructive and destructive interference. In order to prevent these features from masking true atmospheric and surface features, we tested several spectral smoothing scenarios. Based on these tests, we adopted a Gaussian filter with a $10 \mathrm{~nm}$ FWHM applied to all channels below $0.745 \mu \mathrm{m}$ and a $20 \mathrm{~nm}$ FWHM filter for all channels above 0.745 $\mu \mathrm{m}$. The black line in Fig. 8(b) is the sand spectrum after application of the filter illustrating the effec- (a)

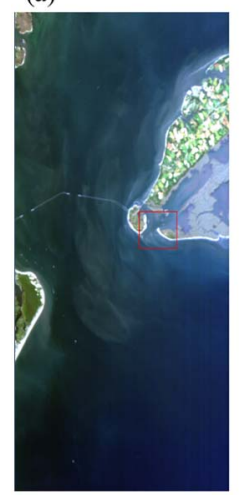

(b)

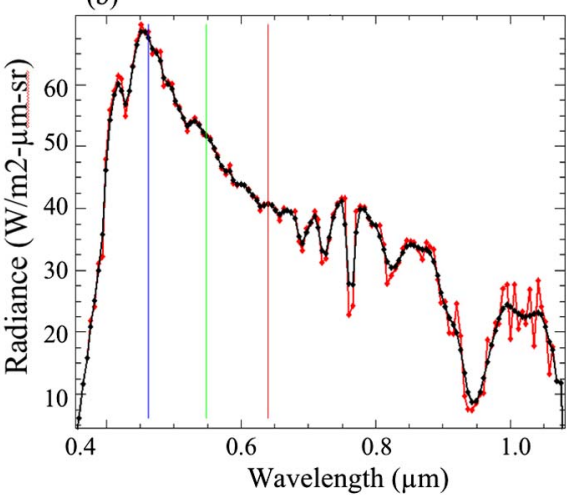

Fig. 8. (Color online) (a) HICO true color image acquired over the mouth of Chesapeake Bay on October 7, 2009; (b) Unsmoothed radiance spectrum (red line) of a sandy pixel and the corresponding smoothed spectrum (black line) of the same pixel. tiveness of the smoothing in removing the high frequency oscillations due to etaloning.

\section{Results and Verifications}

The vicarious techniques described in Section 3 are currently used in routine processing of the $\overline{\mathrm{HICO}}$ data from the L1A DNs to the L1B radiances. Additional results and verification are presented below.

Figure 9(a) is a HICO true color image acquired over Hong Kong and nearby sea areas on October 2,2009 . Figure 9(b) shows examples of radiance spectra over a turbid case 2 water area, green vegetation, and clouds. The shapes of these spectra are quite similar to those of spectra acquired with other instruments, such as the NASA JPL AVIRIS, over similar types of targets. The oscillations resulting from etaloning effects seen in Fig. 1(b) and Fig. 8(b) are significantly suppressed in the Fig. 9(b) spectra.

Figure 10 shows an example of verification of HICO radiometric calibration through comparison of a HICO dataset acquired over the month of the Yangtze River on January 18, 2010, to an Aqua MODIS dataset acquired on the same day only 20 minutes later. The solar zenith angle and view zenith angle for the HICO data were approximately $53 \mathrm{deg}$ and $15 \mathrm{deg}$, respectively. The corresponding angles for the MODIS data were approximately $52 \mathrm{deg}$ and $8 \mathrm{deg}$, respectively. The solar angles and view angles for both datasets were extremely close. This minimizes the possible differences due to bidirectional reflectance factors between the two datasets. This Aqua MODIS dataset acquired was not used in the vicarious calibration process described in Subsection 3B. Figure 10(a) is a HICO true color image. Figure $\overline{10(b)}$ is the corresponding MODIS true color image. Area 1, 2, and 3, marked in both images, contain fairly clear waters, slightly turbid waters, and very turbid waters, respectively. Radiance values from both datasets over the three areas were extracted and plotted in Fig. 10(c), (d), and (e), respectively. Over the clear waters $[\overline{\text { Fig. }} 10(\bar{c})]$ and
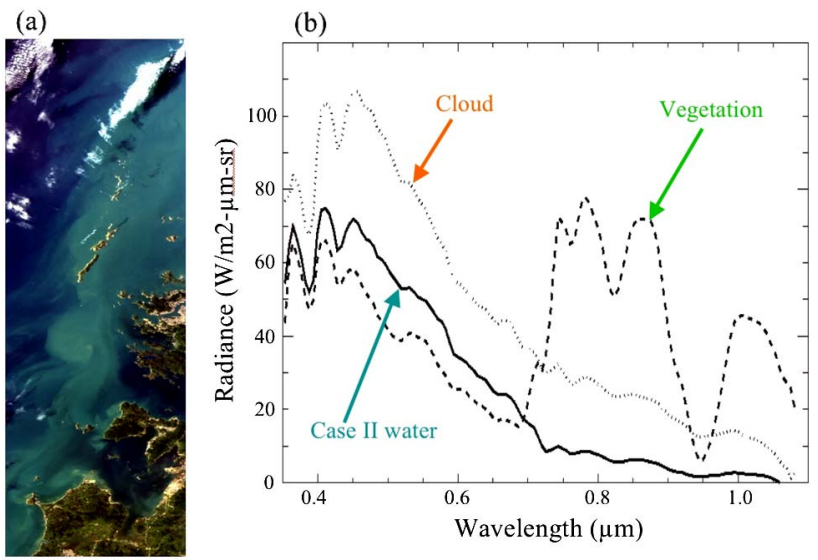

Fig. 9. (Color online) (a) HICO true color image acquired over Hong Kong and nearby water areas on October 2, 2009. (b) Sample Level $1 \mathrm{~B}$ radiance spectra $\left[50 \mathrm{~W} /\left(\mathrm{m}^{2} \mathrm{sr} \mu \mathrm{m}\right)\right]$ over case 2 water, green vegetation, and cloud. 

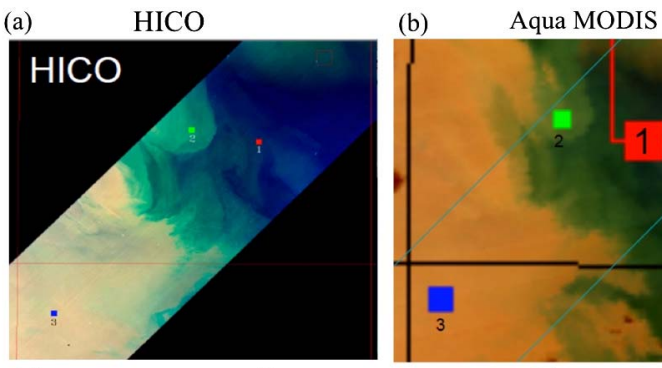

(c)
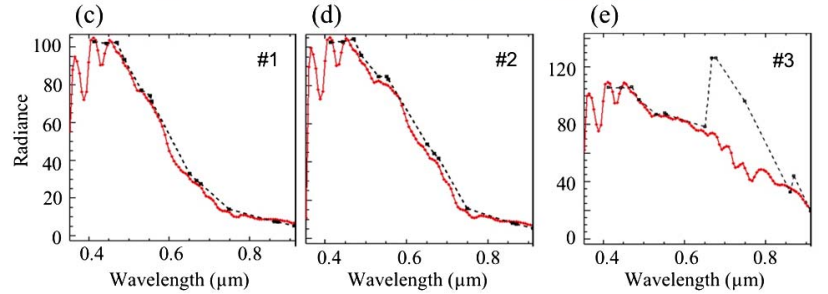

Fig. 10. (Color online) HICO true color image (a) acquired over the mouth of Yangtze River in Eastern China on January 18, 2010, the corresponding Aqua MODIS true color image (b) acquired 20 minutes later on the same day, and comparisons between HICO and MODIS data acquired over clear waters (c), fairly turbid waters (d), and very turbid waters (e).

slightly turbid waters [Fig. 10(d)] and over spectral regions where MODIS has narrow channels, both the MODIS data and HICO data agreed quite well. Specifically, the mean of the differences between HICO and MODIS data pairs for all the nonsaturated MODIS channels below $0.865 \mu \mathrm{m}$ was $4.88 \%$. This verifies the success of our HICO vicarious calibration techniques. Over the very turbid waters [Fig. 10(e)], several MODIS data points also agreed very well with the corresponding HICO data points. However, four MODIS data points were located well above the HICO spectral curve, which is due to saturation of the four MODIS ocean color channels over the very turbid waters. The MODIS ocean color channels were originally designed for remote sensing of case 1 waters $[11,15]$, not for the very turbid and bright case 2 waters.

Figure 11 shows another case of verification of HICO radiometric calibration through comparing a HICO data set acquired over Lake Eyre in Australia at UTC 02:03 on May 11, 2010, with a Terra MODIS dataset acquired slightly less than 1 hour earlier on the same day. The solar zenith angle and view zenith angle for the HICO data were approximately $52 \mathrm{deg}$ and $16 \mathrm{deg}$, respectively. The corresponding angles for the MODIS data were approximately $47 \mathrm{deg}$ and $10 \mathrm{deg}$, respectively. Because the solar angles change slowly around local noon time, the solar angles and view angles for both datasets were also quite close. This Terra MODIS dataset was also not used in the vicarious calibration process (see Subsection 3B). Figure $11(\mathrm{a})$ is a HICO true color image. Figure $1 \overline{1(b)}$ is the corresponding Terra MODIS true color image. Three spatially uniformed areas are marked in letters 1,2 , and 3 respectively on the two images. Area 2 was covered by turbid waters, while Area 1 and 3 were covered by uniform land surfaces. Radiances (a)
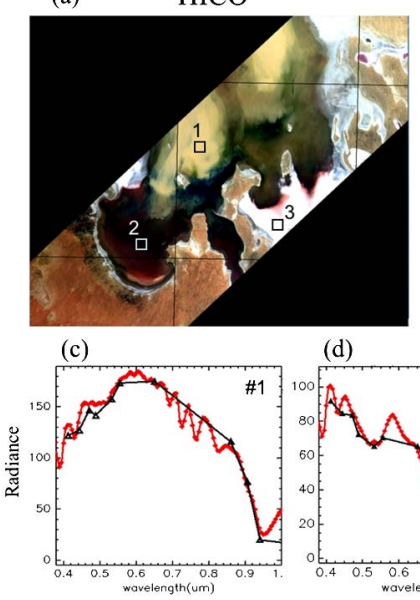

(d)

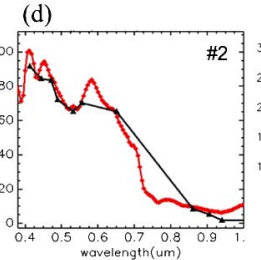

(b) Terra MODIS

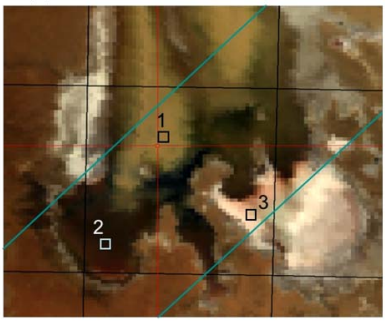

(e)

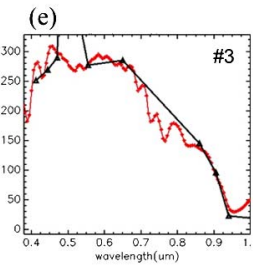

Fig. 11. (Color online) HICO true color image (a) acquired over Lake Eyre, Australia on May 11, 2010, the corresponding Terra MODIS true color image (b) acquired less than 1 hour earlier on the same day, and comparisons between HICO and MODIS data acquired over Area 1, 2, and 3, as marked in (a) and (b).

values from both datasets over the three areas were extracted and plotted in Figs. 11(c), 11(d), and 11(e), respectively. Most of the MODIS data points on the three spectral plots were consistent with those of corresponding HICO data points. The mean of the differences between HICO and MODIS data pairs for those nonsaturated MODIS channels below $0.65 \mu \mathrm{m}$ was $5.54 \%$. This verified again the success of our HICO vicarious calibration techniques. In Fig. 11(e), because a few MODIS ocean color channels saturated over the bright land surface, these points were out of the range of the plot. The HICO channels did not saturate over the bright land surfaces.

\section{Summary}

Because the HICO instrument is not equipped with any onboard spectral and radiometric calibration devices, vicarious calibrations are necessary for spectral and radiometric calibration of HICO data acquired from the ISS. We have developed spectrum-matching techniques for wavelength and spectral resolution calibrations for HICO data by fitting atmospheric gaseous absorption bands and extraterrestrial solar lines. We have developed an empirical technique for correction of second-order light effects for channels above $0.8 \mu \mathrm{m}$ based on the removal of shallow underwater feature artifacts in HICO images for channels above $0.8 \mu \mathrm{m}$. We have also obtained a scaling factor and a scaling curve based on intersatellite data comparisons and on modeling cloud reflectance properties at the TOP. At present, these vicarious techniques are used operationally for converting the HICO L1A DNs to the $\mathrm{L} 1 \mathrm{~B}$ radiances. Improvements to the present algorithms are possible as we gain more in-depth understanding of the HICO laboratory calibration data and the ISS HICO data in the future. 
The HICO instrument was sponsored by the U.S. Office of Naval Research as part of the Innovative Naval Prototype program and built at the NRL. $\mathrm{HICO}$ is integrated and flown under the direction of the DoD Space Test Program. The research reported here was partially supported by the Office of Naval Research.

\section{References}

1. R. L. Lucke, M. Corson, N. R. McGlothlin, S. D. Butcher, D. L. Wood, D. R. Korwan, R. R. Li, W. A. Snyder, C. O. Davis, and D. T. Chen "The hyperspectral imager for the coastal ocean: instrument description and first images," Appl. Opt. 50, 15011516 (2011).

2. M. R. Corson and C. O. Davis, "A new view of coastal oceans from the space station," EOS 92, 161-168 (2011).

3. B. Franz, S. Bailey, P. Werdell, and C. McClain, "Sensorindependent approach to the vicarious calibration of satellite ocean color radiometry," Appl. Opt. 46, 5068-5082 (2007).

4. Etaloning in back illuminated CCDs, Technical Note, Roper Scientific, Inc. (2000), available online at http://www .princetoninstruments.com/Uploads/Princeton/Documents/ Whitepapers/etaloning.pdf.

5. Y. S. Chang and J. H. Shaw, "A nonlinear least squares method of determining line intensities and half-widths," Appl. Spectroscopy 31, 213-220 (1977).

6. B.-C. Gao, M. J. Montes, and C. O. Davis, "Refinement of wavelength calibrations of hyperspectral imaging data using a spectrum-matching technique," Rem. Sens. Environ. 90, 424-433 (2004).

7. R.-R. Li, R. Lucke, D Korwan, and B.-C. Gao, "A technique for removing second-order light effects from hyperspectral imaging data," IEEE Trans. Geosci. Remote Sens. 50, 824-830 (2012)

8. R. O. Green, M. L. Eastwood, C. M. Sarture, T. G. Chrien, M. Aronsson, B. J. Chippendale, J. A. Faust, B. E. Pavri, C. J. Chovit, M. Solis, and M. R. Olah"Imaging spectroscopy and the airborne visible infrared imaging spectrometer (AVIRIS)," Rem. Sens. Environ. 65, 227-248 (1998).

9. D. M. Wieliczka, S.-S. Weng, and M. R. Querry, "Wedge shaped cell for highly absorbent liquids: Infrared optical constants of water," Appl. Opt. 28, 1714-1719 (1989).

10. B.-C. Gao, M. J. Montes, Z. Ahmad, and C. O. Davis, "Atmospheric correction algorithm for hyperspectral remote sensing of ocean color from space," Appl. Opt. 39, 887-896 (2000).

11. V. V. Salomonson, W. L. Barnes, P. W. Maymon, H. E Montgomery, and H. Ostrow, "MODIS: Advanced facility instrument for studies of the earth as a system," IEEE Trans. Geosci. Remote Sens. 27, 145-153 (1989).

12. D. K. Clark, H. R. Gordon, K. J. Voss, Y. Ge, W. W. Broenkow, and C. Trees, "Validation of atmospheric correction over the oceans," J. Geophys. Res. 102, 17209-17217 (1997).

13. E. F. Vermote, D. Tanré, J. L. Deuzé, M. Herman, and J. J. Morcrette, "Second simulation of the satellite signal in the solar spectrum, 6S: An overview," IEEE Trans. Geosci. Remote Sens. 35 675-686 (1997).

14. P. Stamnes, W. H. Knap, R. B. A. Koelemeijer, and N. A. J. Schutgens, "Radiation and cloud studies with GOME in preparation for future spectrometer missions," (2007), available online from http://earth.esa.int/pub/ESA_DOC/gothenburg/ 111stamm.pdf.

15. M. D. King, Y. J. Kaufman, W. P. Menzel, and D. Tanre, "Remote sensing of cloud, aerosol and water vapor properties from the Moderate Resolution Imaging Spectrometer (MODIS)," IEEE Trans. Geosci. Remote Sens. 30, 2-27 (1992). 